\title{
Conclusions
}

10 Comme sa teneur en vitamine $C$, la teneur en vitamine $B_{1}$ et en vitamine $B_{2}$ du lait de vache vendu dans une même laiterie parisienne est assez variable d'un jour à l'autre ;

20 L'actinisation du lait provoque des pertes moyennes de ces deux vitamines'B qui sont respectivement de l'ordre de $16 \%$ et de $10 \%$.

(Travail du Laboratoire de Physiologie de la Nutrition de l'Ecole des Hautes Etudes et du Centre de Recherches sur l'Alimentation de l'Institut National de la Recherche Agronomique.)

\section{DÉTERSIFS ET DÉSINFECTANTS (1)}

$$
\text { par }
$$

\section{E. R. HISCOX et A. ROWLANDS}

Institut National de Recherches de Laiterie de Shinfield (près Reading)

Une note [1] indique en termes simples le rôle, la composition, la préparation et l'utilisation des détersifs. Des renseignements d'un caractère analogue ont été donnés par MoDowall [2], PARKER [3] et RAHN [4].

Les méthodes d'appréciation des détersifs continuent à attirer l'attention. LIDDIARD et HARWOOD [5] ont imaginé une méthode électrique pour mesurer la facilité de rinçage des détersifs sur des surfaces de verre. Un morceau de verre de $5 \times 2 \times 0,5 \mathrm{~cm}$. serré entre deux électrodes et relié à un milliampèremètre, sous une différence de potentiel de $7 \mathrm{~V}$. est immergé dans la solution détersive et la différence dans le courant passant après immersion pendant une seconde dans des lots successifs d'eau de rinçage, indique la proportion d'alcali restant dans la pellicule. Des solutions à $5 \%$ de $\mathrm{Na}^{2} \mathrm{CO}^{3}$ et de $\mathrm{NaOH}$ exigèrent respectivement quatre et neuf rinçages ; l'addition de $1 \%$ d'hexamétaphosphate de soude du commerce à $\mathrm{NaOH}$ réduisit le nombre de rinçages nécessaire à cinq. DAVIS, WARD et LIDDIARD [6] ont décrit un instrument, qui est une modification du pont de Wheatstone, pour mesurer la concentration des détersifs dans les machines à laver les bouteilles. Des tests d'évaluation de l'efficacité de nettoyage des détersifs ont été imaginés par Gilcreas et O'Brien [7], Hughes et Bernstein [8]. Des lames porte-objets de microscope ou des plaques carrées de verre laminé d'épaïsseur uniforme entièrement nettoyées furent, après traitement par un mélange salissant synthétique, lavées

(1) E. R. Hiscox et A. Rowlands. Revue des progrès de la Seience laitière. Extrait de The Journal of Dairy Research, 1947, XV, 204-208. Traduetion de C. Wolf. 
mécaniquement dans des conditions déterminées dans des solutions aqueuses de détersifs, amenées à un degré donné de dureté, et on mesura la pellicule résiduelle. GILCREAS et O'BRTEN utilisèrent un colorimètre photométrique pour mesurer la pellicule résiduelle, son intensité étant exprimée par la différence de transmission de lumière entre les plaques-témoins propres et les plaques salies puis nettoyées. Pour obtenir de plus grandes différences de transmission de lumière entre les plaques propres et celles ayant une couche résiduelle, les plaques furent traitées avec du charbon activé avant la notation des résultats. Hughes et BERnsterin mesurèrent la proportion de lumière incidente dispersée par la pellicule résiduelle. Pour cela, ils prirent des dispositions pour que la lumière de rayons parallèles d'intensité constante soit empêchée d'atteindre la surface active d'une cellule photoélectrique, une proportion seulement de la lumière qui est dispersée quand les carrés de verre sont introduits dans le faisceau lumineux pouvant l'atteindre, les résultats étant notés en termes de déviation du galvanomètre. Des méthodes de laboratoire imitant les conditions pratiques d'évaluation de l'efficacité germicide des détersifs ont été imaginées par Mewes [9]. Des bouteilles sales furent lavées dans conditions déterminées et le nombre de colonies par centimètre carré se développant dans un milieu de gélose étalé sur la surface interne utilisé pour évaluer l'efficacité comparative de divers détersifs. De petits morceaux de fer-blanc salis et lavés dans des conditions déterminées furent utilisés pour mesurer l'aptitude des détersifs au nettoyage des boîtes à lait; le nombre d'organismes survivants fut déterminé soit par une méthode de rinçage, soit par des fixations à la surface de plaques de gélose.

Des tests par Hоввs et WiLson [10] de la destruction de Bact. Coli et d'un organisme sporogène par le $\mathrm{NaOH}$ ont prouvé que la vitesse de désinfection n'était pas influencée d'une façon notable par la présence de $0,1 \%$ de lait, résultat qui est en contradiction avee celui trouvé par MatTick et SHARPE [11] qui indiquent que la présence de lait amenait une diminution considérable de l'indice $K$. Pour éviter la difficulté provenant des variations de la vitesse de réaction qui peuvent se produire au cours de la désinfection, Новвs et WiLson [10] proposent que $K$ soit caleulé pour le stade moyen de la réaction et Withell [12] sùggère que le temps pris pour détruire $50 \%$ des organismes soit choisi comme mesure comparative de l'efficacité bactéricide. Des résultats basés sur la destruction de $25 \%$ des spores de B. subtilis furent utilisés par HоBBS et Wuson [13] dans l'établissement d'un tableau de combinaisons de la concentration en $\mathrm{NaOH}$, de durée de contact et de température convenant pour le nettoyage des bouteilles de lait. La concen- 
tration en $\mathrm{NaOH}$ indiquée est plus forte que celle trouvée efficace dans la pratique du nettoyage à la machine des bouteilles par Matтiok et Hox [14]. Il a été souligné [1] que les résultats de tests de laboratoire, surtout quand on utilise des spores extrêmement résistantes, peuvent n'avoir que très peu de rapport avec l'efficacité germicide des détersifs dans la pratique, si la majorité des organismes à détruire est dan's le stade végétatif, car, ainsi que l'ont montré HoBBs et WrLson [13], la valeur de $K$ d'une solution à $0,5 \%$ de $\mathrm{NaOH}$ est environ 3 millions de fois plus grande pour les spores de Bact. Coli que pour celles de B. subtilis.

Ames et Smith [15] ont trouvé, lors d'expériences effectuées par eux sur la destruction de Bact. Coli dans l'eau en présence de 0,25\% d'azote, que, même entre $10^{\circ}$ et $40^{\circ} \mathrm{C}$., la vitesse de désinfection par le chlore était notablement influencée par la température. WEBER et LEVINE [16] en utilisant la durée nécessaire pour détruire $99 \%$ des spores de $B$. metiens trouvèrent qu'en doublant la concentration du chlore, on réduisait la durée du temps nécessaire à la destruction d'environ $50 \%$; une diminution de température de $10 \%$ amena une augmentation du double de la durée du temps nécessaire à la destruction.

Pour éviter la recontamination des bouteilles qui, dans des machines fonctionnant convenablement sont habituellement stérilisées d'une façon satisfaisante dans la solution détersive, Новвs et WiLson [13] préconisent un rinçage chaud à une température pas inférieure à $48^{\circ} 8$ pour enlever le détersif, les bouteilles étant ensuite refroidies par des rinçages externes. Crowther et BERRY [17] conseillent l'utilisation du chlore maintenu à une concentration de 50 pour mille dans le rinçage chaud qu'on fait recirculer, quoique HoвBs et WiLson [13] aient souligné la difficulté de vérifier le dosage quand la teneur de l'eau en matière organique varie d'heure en heure ainsi que c'est le cas dans les machines à laver les bouteilles.

La destruction des streptocoques de la mastite pénétrant dans l'eau utilisée pour laver les mamelles par 200 pour mille de chlore actif a été confirmée par Bryan, Darby, MaLmann et Corbett [18]; mais à ces concentrations les solutions perdent rapidement leur efficacité en raison de la présence de matières organiques. Des solutions de savon et d'alcools sulfonés furent inefficaces eontre de nombreuses souches de streptocoques, Staph. aureus et Bact. Coli. Byers et EwaLt [19] ont trouvé qu'en trempant les gobelets trayeurs et en lavant les mamelles dans de l'eau contenant 150 pour mille de chlore actif, on réduisait la numération des colonies du lait frais de $34 \%$; après le lavage de sept vaches, la teneur en chlore n'était plus que de 50 pour mille.

En Angleterre et dans le pays de Galles, l'interdiction d'utilisa- 
tion des hypochlorites pour la stérilisation des ustensiles de laiterie autres que les machines à traire fut abandonnée en raison des circonstances de guerre. Ne sont autorisées que les solutions d'hypochlorite de sodium de concentrations approuvées et ne contenant pas moins de $0,7 \%$ de chlorate de sodium pour permettre de les déceler si elles sont ajoutées volontairement au lait. On a trouvé que de petites additions d'hypochlorite de sodium au lait oxydent presque complètement l'acide ascorbique [20]. BARKworTH [21] a montré que la durée de réduction du bleu de méthylène d'échantillons de lait peut être augmentée par l'addition d'hypochlorite au lait, augmentation variant avec différents échantillons de 0 à 5 heures et de 0 à 3 heures quand on ajoutait respectivement 180 et 10 pour mille de chlore actif. HAUSER et KING [22] en utilisant la méthode de numération des colonies, ont trouvé que l'addition de 10 pour mille de chlore actif était manifestement sans effet sur la vitesse de la croissance bactérienne dans le lait cru et pasteurisé conservé à $+10^{\circ} \mathrm{C}$. ; lá vitesse de croissance fut légèrement retardée avec 50 pour mille et encore plus avec 100 pour mille. Avec 10 pour mille, le temps pris par le lait eru pour sûrir à $T 10^{\circ} \mathrm{C}$. augmenta de 4 à 7 jours.

Escomptant l'utilisation plus générale des hypochlorites et comprenant l'importance de leur utilisation correcte, Mațiok, Hoy et Neave [23] ont donné des détails sur des méthodes convenant d'une façon générale pour les ustensiles de la ferme et de la laiterie. Ils recommandent une concentration de 250 à 300 pour mille de chlore actif, et insistent sur l'importance de leur application à des surfaces entièrement nettoyées et sur une durée de contact suffisante. Le brossage est recommandé, ou la pulvérisation dans le cas de grandes surfaces, avec une solution d'hypochlorite et $0,25 \%$ de carbonate de soude ou d'un autre détersif à une température pas inférieure à $43^{\circ} 3 \mathrm{C}$. après un rinçage complet préliminaire dans l'eau froide ou chaude pour enlever les résidus lactés. L'eau utilisée pour le rinçage final devrait contenir 10 à 50 pour mille de chlore actif quand il y a un doute sur sa pureté. On recommande la stérilisation une fois par semaine à la vapeur ou à l'eau bouillante, surtout pendant la saison chaude. Dans des essais effectués en utilisant ces méthodes, mais avec des solutions d'hypochlorite de soude froides, dans deux fermes accréditées (pour la production de lait de qualité supérieure) les uns avec des machines à traire et les autres par des traites manuelles, Rowlands [24] obtint de bons résultats au début de l'année quand le temps était frais ; mais rencontra des difficultés pour répondre aux exigences des fermes accréditées avee l'arrivée de la saison chaude. En faisant bouillir les ustensiles une fois par semaine, on ne rencontra aucune autre difficulté excepté pendant 
une semaine extrêmement chaude en août. Phillips et Thomas [25] obtinrent des résultats légèrement meilleurs en passant à la vapeur des unités de machine à traire avec un jet de vapeur pendant 3 minutes, puis en trempant les unités entre les traites dans une saumure d'hypochlorite contenant 200 pour mille de chlore actif ; mais les deux méthodes furent supérieures au trempage des unités dans une solution de métasilicate de sodium à $0,5 \%$ (gobeletstrayeurs en alliage d'aluminium). Dans des essais ayant duré une année entière dans deux fermes, les résultats bactériologiques des essais des laits furent constamment excellents quand les unités, après lavage dans l'eau et un alcali très chaud, furent trempées entre les traites dans une solution contenant 200 pour mille de chlore actif ; quand les unités étaient seulement rincées avee des solutions d'hypochlorite de la même concentration, la numération géométrique moyenne des échantillons de lait pendant juillet et août dépassa 500.000 par $\mathrm{ml}$. JoHNs [26] continue à recommander le traitement des eaoutchoucs de machines à traire avec une solution froide de $\mathrm{NaOH}$ à $0,5 \%$ et déclare qu'un traitement de 5 minutes avec une solution de cette force est suffisant pour les maintenir dans un état hygiénique. Dans une étude de méthodes de nettoyage des machines à traire ayant duré plus de deux ans $[27,28,29]$ on a trouvé que les numérations des colonies d'échantillons de lait étaient plus basses quand les unités étaient remplies avec une solution de $\mathrm{NaOH}$ à $0,5 \%$ entre les traites et démontées une fois par semaine que quand elles étaient démontées journellement ; la première méthode permit une économie hebdomadaire de 30 à 60 minutes par machine de 2 unités. Les numérations de colonies furent, chose surprenante, plus basses pendant le trimestre septembre-novembre que pendant le trimestre décembre-février.

En Nouvelle-Zélande, les machines du type à valve sont utilisées d'une façon générale. Pour éviter les déficiences de combustible qui pourraient rendre impossible la méthode officielle qui consiste à rincer chaque unité avec 18 litres d'une solution bouillante de $\mathrm{NaOH}$ suivie par 4 1. 5 d'eau bouillante, les rinçages à la soude et à l'eau bouillante peuvent être réduits respectivement à 41.5 et à 2 1. 25 si les machines sont d'abord entièrement rincées avec de l'eau froide [30]. Des méthodes analogues à celles utilisées en NouvelleZélande sont préconisées en Australie [31]. MoIR [32] a insisté sur l'importance de l'utilisation de $\mathrm{NaOH}$ quand le volumé d'eau bouillante est insuffisant. Un rapport préliminaire [33] sur des essais de la méthode d'immersion utilisant du $\mathrm{NaOH}$ à $0,5 \%$ indiquait que si ce traitement peut convenir pour les manchons et les tuyauteries à lait, d'autres méthodes sont nécessaires pour la conduite principale et la valve ; leur traitement au $\mathrm{NaOH}$ chaud 
et un rinçage avec une solution d'hypochlorite donnèrent des résultats prometteurs. SHERMAN [34] a trouvé qu'un rinçage avec 100 pour mille de chlore actif immédiatement avant la traite donnait de meilleurs résultats que l'immersion des parties en caoutchouc dans la solution de chlore entre les traites.

La conservation du caoutchouc a pris une très grande importance pendant les années de guerre. Morr [35] a observé que des manchons de gobelets-trayeurs peuvent être rendus de nouveau utilisables par une ébullition prolongée dans une forte solution de $\mathrm{NaOH}$. Les manchons contenaient en moyenne $10 \%$ en poids de matière grasse ; dans quelques parties, la matière grasse atteignait $30 \%$. Kristensen [36] a trouvé que les caoutchoucs régulièrement traités par rinçage avec une solution bouillante de $\mathrm{NaOH}$ sont maintenus plus exempts de matière grasse que quand on utilise $\mathrm{Na} 2 \mathrm{CO}^{3}$.

Les pots qui sont inefficacement layés et stérilisés dans les laiteries peuvent contenir d'innombrables bactéries quand ils arrivent dans les fermes où, par conséquent, leur stérilisation suffisante peut être difficile s'il n'y a pas de vapeur disponible. L'adoption du "National Milk Testing and Advisory Scheme" (Plan National d'examen du lait et de consultation) a attiré l'attention sur le problème et pour orienter les laiteries, le Ministère de l'Agriculture et des Pêches a publié des notes traitant des méthodes de lavage manuel [37] et du fonctionnement des machines à laver les pots [38]. Quand on pratique le lavage manuel, le passage à la vapeur des pots et des couvercles pendant 2 minutes est recommandé pour obtenir des pots secs et satisfaisants au point de vue bactérien. Si on veut que l'opération soit efficace, le maintien d'une température convenable dans les diverses parties des machines à laver est considéré comme essentiel et un séchage final à l'air chaud doit assurer le séchage des pots. Il est signalé que d'excellents résultats peuvent être obtenus sans utilisation de détersifs. Thomas, Dixon et Jones [39] ont trouvé que, dans beaucoup de laiteries du pays de Galles, les pots après un lavage manuel sont passés à la vapeur pendant seulement 10 à 30 secondes, et que, par conséquent, les résultats bactériologiques des rinçages étaient défectueux. L'importance du passage à la vapeur des couvercles aussi bien que des pots a été confirmée par JAMIESON et CHEN [40]. Les résultats obtenus par Bowyer, Fischer, Cooper-Willis, Davis et MiCHAELIS [41] quand les pots et les coucervles sont remplis avec une solution contenant 250 pour mille de chlore actif et vidés après 130 secondes, ne furent pas aussi bons que le passage à la vapeur pendant 30 secondes quand les pots sont examinés dans la demiheure qui suit le lavage ; mais après 24 heures, ils étaient légèrement 
meilleurs, les traces de chlore laissées dans les pots exerçant un effet retardateur sur la multiplication bactérienne. Dans leurs expériences, ils trouvèrent des pots non usuels qui furent plus difficiles à stériliser. Le même phénomène a aussi été observé par d'autres expérimentateurs et RowLANDS [42] a montré qu'il n'est pas du tout en rapport avec la qualité bactériologique du lait cru contenu dans les pots avant lavage. NEAve [43] a montré que l'état des ustensiles a une influence importante sur l'efficacité de la stérilisation par les hypochlorites. Avec des pots exempts de rouille, $92 \%$ donnèrent des résultats bactériologiques satisfaisants quand, wà la suite du lavage, ils furent traités avec une solution contenant 200 pour mille de chlore actif, tandis que sur ceux ayant des surfaces rouillées, seulement $75 \%$ donnaient des résultats satisfaisants ; quand un traitement similaire fut appliqué à des pots qui avaient contenu du lait acide, les pourcentages satisfaisants furent respectivement 44 et 14 .

NFAvE [44] obtint d'excellents résultats avec des pots lavés dans un laveur mécanique rotatif actionné manuellement. qui paraissait être hors de service. En lavant 2 à 3 pots par minute, et en prenant soin de maintenir le détersif à une concentration équivalente à $0,5 \%$ de $\mathrm{NaOH}$ tout en maintenant une température suffisamment élevée dans les diverses parties, les résultats, même après 28 heures à la température ambiante, furent satisfaisants. Crowther et Berry [17] sont en faveur de l'utilisation de l'hypochlorite à une concentration de 300 pour mille de chlore actif dans la partie des laveurs mécaniques où s'effectue le rinçage fínal très chaud, sinon on obtient des résultats défectueux. Il n'est pas donné de renseignements sur l'effet corrosif possible de telles solutions, quoiqu'il soit évident que non seulement les pots, mais aussi les pompes et les cuves puissent être attaqués par de telles concentrations.

L'utilisation de vapeur acidifiée pour le traitement final des pots a été très étudiée en Amérique. D'après Parker [45, 46], quand des alcalis sont utilisés dans les cuves de détersifs des machines à laver les pots, l'accumulation des protéines dans la solution produit le dépôt d'une pellicule sur la surface des pots, ce qui, malgré le maintien de la température à $76^{\circ} 7 \mathrm{C}$, ou plus, et le maintien de l'alcalinité à $0,25 \%$ de $\mathrm{Na}^{2} \mathrm{O}$, produit des difficultés croissantes dans l'obtention de pots stériles au fur et à mesure de la progression de l'opération. On a trouvé que la flore de tels pots consiste surtout en organismes Gram négatifs, les types protéolytiques et oxydants des genres Pseudomonas et Achromobacter étant prédominants. Le traitement final des pots avec de la vapeur chargée en acide gluconique de concentration suffisante pour réduire. le 
$p \mathrm{H}$ de la surface du pot à 6,0 à 6,05 donna des numérations inférieures et supprima la flore Gram négative. Des résultats encore meilleurs furent obtenus quand l'alcali était remplacé par un mélange du commerce (spécialité), se composant d'acides organiques, d'agents mouillants et d'un produit empêchant la corrosion. Les résultats d'une comparaison de détersifs acides et alcalins dans des laveurs mécaniques de pots sont aussi donnés par ScHWARzKOPF $[47,48]$ et SCALES $[49,50]$. Tous deux sont d'accord pour indiquer que les détersifs acides donnent de meilleurs résultats, sont moins coûteux et moins corrosifs que les détersifs alcalins maintenus à une concentration équivalente à environ $0,05 \%$ de $\mathrm{NaOH}$.

Beaucoup de grosses pièces de matériel utilisées pour le traitement du lait ne sont pas facilement stérilisées par la vapeur, et des méthodes alternatives possibles pour le maintien de leur état hygiénique ont été exposées [51]. NEAvE et Hox [52] ont pu stériliser de grandes cuves en acier inoxydable bien nettoyées et à surfaces lisses en les pulvérisant avec 1 1. 222 par mètre earré de surface d'une solution froide à 200 pour mille de chlore actif et à $0,25 \%$ de carbonate de soude. On a trouvé qué les coussinets et les surfaces rugueuses sont difficiles à stériliser par cette méthode. Aux Etats-Unis [53], on recommande la pulvérisation pendant au moins 5 minutes d'une solution ne contenant pas moins de 250 pour mille de chlore actif et on insiste sur la nécessité d'un nettoyage et d'une stérilisation convenable des citernes automobiles et des citernes sur wagons, aussi bien aux laiteries de remplissage qu'à celles de vidange.

Des agents actifs de la surface sont incorporés dans quelques détersifs commerciaux (spécialités) utilisés pour le nettoyage du matériel laitier ; mais on ne possède que très peu de renseignements sur leur efficacité dans la pratique. La valeur des combinaisons quaternaires d'ammonium pour la stérilisation du matériel laitier a été l'objet de l'attention de quelques chereheurs américains. RAHN [4] a montré que, comme pour le chlore, il y a quelque perte d'efficacité en présence de matière organique, et que leur efficacité bactéricide est très réduite si elles sont appliquées à du matériel qui n'est pas complètement lavé. Krog et MARshall [54] ont trouvé que l'utilisation de chlorures d'alkyldiméthylbenzyle d'ammonium à une concentration de 1 pour 6.000 (170 pour mille) pour le rinçage final d'un grand pasteurisateur réduisait de 60 à $98 \%$ le nombre de bactéries retrouvées par une méthode de cultures par balayage. En utilisant la même combinaison à une concentration d'environ 800 pour mille pour pulvériser des pots à lait lavés, avant leur utilisation dans les fermes, JAMIESON et CHEN [55] ont obtenu une 
réduction de 96 à $99 \%$ du nombre de bactéries décelables par une méthode d'ensemencement par balayage ; une réduction analogue fut obtenue en pulvérisant les pots avec une solution d'hypochlorite contenant environ 183 pour mille de chlore actif. Des tests effectués par Frayer [56] en utilisant un ensemencement d'environ 5 millions de cellules viables par $\mathrm{ml}$. de cultures âgées de 24 heures sur bouillon de Bact. Coli, Bact. aerogenes et B. subtilis dans des solutions de ce germicide dans l'eau ont prouvé qu'à une concentration de 400 pour mille il n'y avait pas de survivants après 15 secondes de contact avec Bact. aerogenes et B. subtilis et après 30 secondes avec Bact. Coli ; en utilisant des solutions contenant 200 pour mille il n'y avait pas de survivants après 1 minute avec Bact. aerogenes, et après 3 minutes avee Bact. Coli et $B$. subtilis. VALKo et DU BoIs [57] ont montré que l'action antibactérienne de cations actifs de la surface peut être inversée dans certaines conditions par des agents actifs des anions. En utilisant du sulfate dodécyle de sodium comme agent antagoniste, ils ont trouvé qu'il était possible d'inverser l'action d'une dilution à 1 pour 3.000 (330 pour mille) d'un mélange de chlorures d'alkyldiméthylbenzyle d'ammonium après action d'une culture âgée de 22 à 26 heures de Staph. aureus pendant 10 minutes, mais pas après 30 minutes. Il n'a probablement pas été tenu compte de ce facteur par les chercheurs étudiant les questions de laiterie, quoique JAmeson et CHen aient affirmé que, dans leur étude, le transfert immédiat de balayages dans $9 \mathrm{ml}$. de lait écrémé stérile minimisait l'effet de passage du germicide. LAWRENCE [58] a rédigé une revue des études sur l'utilisation des combinaisons quaternaires d'ammonium en laiterie.

\section{BIBLIOG RAPHIE}

[1] Minist. Agrie. Fish. (1944). Form., n ${ }^{0}$ B $570 /$ TPB.

[2] Mc Dowall (1941). N.-Z. Journ, Sci. Teeh., 23 A, 146.

[3] Parker (1943). Food Industr., 15, 7, 78; 8, 71; 9, 66.

[4] Rahn (1945). Milk. Pl. Mon., 34, 12, 24.

[5] LTDDTARD et HARWOOD (1944). Dairy Industr., 9, 488.

[6] Davis, Ward et Liddiard (1944), Abstr. Proc. Soc. Agric. Baet., p. 53.

[7] Gilcreas et O'Brien (1941). Amer. Journ. Publ. Hlth., 31, 143.

[8] Hughes et Bernstein (1945). Industr. Engng. Ohem. (Industr. ed.), 37,170 .

[9] Meewes (1941). Z. Hyg. Infelet, Kr., 123, 239 ; Dairy Sci, Abstr., 5, 34, 1943.

[10] Hoввs et Wilson (1942), Journ. Hyg., Oamb., 42, 436.

[11] Mattick et Sharpe (1939). Abstr. Proc. Soc. Agric. Bact,, p. 38.

[12] Withell (1942), Journ. Hyg., Camb., 42, 124, 339.

[13] Новвs et Wilsox (1943). Journ. Hyg., Camb., 43, 96. 
[14] Mattick et Hox (1937). Bottle Washing and Bottle Washing Machines

(Nettoyage des bouteilles et machines à nettoyer les bouteilles)

Shinfield, England ; National Institute for Research in Dairying.

[15] Ames et Sмiтh (1944). Journ. Bact., 47, 445, A 18.

[16] Weber et Lévine (1944). Amer. Journ. Publ. Hlth., 34, 719.

[17] Crowther et BerRy (1944). Dairy Industr., 9, 546.

[18] Bryan, Darby, Mallmann et Cornetr (1942. Journ. Milk Tech., 5, 77.

[19] Byers et Ewalt (1943). Journ. Dairy Sei., 26, 277.

[20] National Institute for Research in Dairying Report (1941-1943), p. 30.

[21] BARKWORTH (1942). Journ. Hyg., Camb., 42, 334:

[22] HaUser et King (1945). Journ. Ass. Off. Agrio. Chem. Wash., 28, 417.

[23] Mattick, Hoy et Neave (1942). The Sterilization of Milk Vessels by

Chlorine in Emergencies (Stérilisation des récipients à lait par le

chlore en cas d'urgence). Shinfield, England : National Institute

for Research in Dairying.

[24] Rowlands (1943). Shinfield, England: National Institute for Research in Dairying. Unpubl. data. (renseignements non publiés).

[25] Phillips et Thomas (1932). Welsh. Journ. Agric., 17, 139.

[26] JoHns (1944). Journ. Milk Tech., 7, 17.

[27] (1941). Rep. Ariz. Agric. Exp. Sta., p. 52.

[28] (1942). Rep. Ariz. Agric. Exp. Sta., p. 49.

[29] (1943). Rep. Ariz. Agric. Exp. Sta., p. 47.

[30] (1944). N.-Z. Journ. Dep. Agric., 69, 421.

[31] (1945). Journ. Dep. Agric. Vict., 43, 121.

[32] MoIr (1944). N.Z. Journ. Dep. Agric., 69, 417.

[33] (1945). Ann. Rep. Sci. Industr. Res. N.-Z., 19. 10.

[34] Sherman (1941). Journ. Aust, Inst. Agric. Sci., 7, 111.

[35] Moir (1943). N.-Z. Journ. Agric., 67, 153.

[36] Kristensen (1944). N.-Z, Journ. Agric., 69, 419.

[37] Minist. Agric. Fish. (1942). Keep those ehurns clean (Maintenez vos pots propres).

[38] Minist. Agric. Fish. (1943). Form., no C 184/TPY.

[39] Thomas, Dixon et Jones (1943). Welsh Journ. Agric, , 17, 131.

[40] Jamieson et Chen (1943). Canad. Dairy Ice Cr. Journ., 22, 1, 21 ; 2,48 .

[41] Bowyer, Fischer Cooper-Willis, Davis et Micharlis (1)45. Abstr. Proc. Sac. Appl. Bact., p. 57.

[42] Rowlands (1944). Shinfield, England: National Institute for Research in Dairying. Unpl. data. (renseignements non publiés).

[43] Neave (1944). Agr. Progr., 19, 28.

[44] Neave (1943). Abstr. Proc. Soc. Agric. Bact., 0. 39.

[45] Parker (1942). Journ. Milk Tech., 5, 37.

[46) PARKer (1943). Industr. Engng. Chem. (Industr. ed.), 35, 100.

[47] Schw Arzkopf (1942). Milk Pl. Mon., 31, 1, 50.

[48] Schwarzkopf (1943), Milk Pl. Mon., 32, 3, 51.

[49] Scales (1942). Milk Dir., 32, 2, 30.

[50] Scales (1942). Food Industr., 14, 4, 41.

51] Minist: Agric. Fish. (1944). Form., no B 557/TPB. 
[52] Neave et Hox (1944). Dairy Industr., 9, 860.

[53] (1943). Journ. Milk Teeh., 6, 101.

[54] Krog et Marshall (1942). Journ. Milk Tech., 5, 343.

[55] Jamieson et Chen (1944). Journ. Milk Tech., 7, 136.

[56] Frayer (1944). Bull. Vt Agric. Exp. Sta., no 511.

[57] VALKo et DU Bors (1944). Journ. Bact, 47, 15.

[58] Lawrence (1945). Ann. Rep. N.. Y. St. Ass. Milk Sanit., 19, 177.

\section{BIBLIOGRAPHIE ANALYTIQUE}

\section{LES LIVRES}

218 Rapport du Laboratoire d'Essais. Lund Aage. Maelkens Indhold af A. Vitamin og Karotin (Teneur du lait en vitamine A et en carotène), p. 115-130. Edité par le Comité National pour l'Entretien des Animaux domestiques. 1 br. de 133 p. Copenhague, 1945. En vente chez Ejvind Christensen, 60 Vesterbrogade Copenhague V. Prix : 1 couronne dan. 50 ore.

Pendant deux périodes d'hiver et pendant l'été les séparant (novembre 1942 à mai 1944), on a analysé par des méthodes chimiques le lait de trois groupes de vaches (du domaine de "Faurholm " près d'Hillerod) pour sa teneur en vitamine A et en carotène à intervalles d'à peu près un mois. La ration d'hiver consistait pour un groupe d'ensilage A. I. V. de luzerne, de betteraves, de coneentrés, de paille et d'un peu de foin. Les deux autres groupes ne reçurent pas d'ensilage, un de ces groupes recevant des betteraves à sucre, l'autre des rutabagas, comme aliments savoureux. Pendant l'été, jes trois groupes étaient sur le même pâtúrage.

Pendant la période d'été, l'alimentation de tous les groupes était riche en carotène ; pendant la période d'hiver, la teneur en carotène de l'alimenta. tion des groupes ne recevant pas d'ensilage était très faible.

Dans le groupe recevant de l'ensilage, la teneur en vitamine par $100 \mathrm{~cm}^{3}$ de lait resta constante (environ $60-80$ unités) pendant toute la période d'hiver, et la teneur en caritène ne diminue que lentement de sorte que l'effet total en vitamine A à la fin de la période d'hiver était de près de 100 unités par $100 \mathrm{~cm}^{3}$ de lait. Dans les groupes alimentés sans ensilage, la teneur en vitamine A diminua pendant la période d'hiver et pendant les derniers mois (mars, avril) cette teneur était d'environ 40 unités avec de très faibles quantités de carotène. Ce n'est que quelques jours après la mise au pâturage que la teneur en vitamine $\mathrm{A}$ et en carotène a augmenté notablement et cette teneur est restée à ce niveau plus élevé jusqu'à ce que les vaches reviennent de nouveau aux rations d'hiver.

Exprimée en unités par gramme de matière grasse de beurre, la valeur totale de la vitamine A a été d'environ 40 unités en été. Pendant la période d'alimentation hivernale, cette valeur a diminué jusqu'à 10-12 unités par gramme de matière grasse du beurre pour les vaches ne recevant pas d'ensi- 